\title{
Determination of molecular weight of heparin by size exclusion chromatography with universal calibration
}

\author{
X. Guo, ${ }^{\mathrm{a}}$ M. Condra, ${ }^{\mathrm{a}}$ K. Kimura, ${ }^{\mathrm{a}}$ G. Berth, ${ }^{\mathrm{b}}$ H. Dautzenberg, ${ }^{\mathrm{b}}$ and P.L. Dubin ${ }^{\mathrm{a}, *}$ \\ ${ }^{a}$ Department of Chemistry, Indiana University-Purdue University, 402 N. Blackford Steet, Indianapolis, IN 46202-3274, USA \\ b Max Planck Institute for Colloid and Interface Research, D-14424 Potsdam, Germany
}

Received 10 June 2002

\begin{abstract}
The molecular weight (MW) of heparin can be accurately determined by size exclusion chromatography using "universal calibration." A universal calibration curve was constructed for Superose 12 with standard pullulan samples and verified using characterized ficoll fractions. This calibration yielded the correct MW of heparin as determined by light scattering, when the ionic strength of the mobile phase was maintained over 1.0 M. Sodium poly(styrenesulfonate) samples were not suitable standards because of adsorption at high salt concentration and repulsion from the packing material at low ionic strength. The extraordinarily high charge density of heparin leads to the need for high salt concentration to screen such repulsions.
\end{abstract}

(c) 2003 Elsevier Science (USA). All rights reserved.

Heparin is a glycosaminoglycan component of the proteoglycans secreted by the mast cells of mammals and is found in the tissues of lymph nodes, skin, intestines, and lungs [1]. Heparin is a highly negatively charged sulfated polysaccharide whose structure is extremely complex: Fig. 1 identifies the structure of its component disaccharides, from 10 to 50 of which comprise most heparin samples [1,2]; it is noteworthy that the number of possible disaccharides is very large. A further complication is that the sequence and the sequence distribution of these disaccharides within the heparin chain is neither fully elucidated nor fully correlated with biological activity.

The physiological activity of heparin is diverse and complex. Historically, it is best known as an anticoagulant, functioning by binding to antithrombin III, which decreases the activation of thrombin and therefore the ability of blood to clot $[2,3]$. Purification and fractionation of clinical heparin alters its molecular weight (MW), "standard heparin" having a molecular weight of about $12 \mathrm{kDa}$, and "low-MW heparin" having a molecular weight of 3-8 $\mathrm{kDa}$ [4]. Low-molecular-weight heparins can be derived by physical, chemical, or enzy-

\footnotetext{
${ }^{*}$ Corresponding author. Fax: 1-317-274-6879.

E-mail address: dubin@chem.iupui.edu (P.L. Dubin).
}

matic degradation of commercial heparin [1]. While it is generally agreed that heparin samples of different MW have different biological activities, there is no obvious relationship between MW and activity in general, enhanced activity being correlated with increased MW in some cases and with decreased MW in others $[5,6]$. This may be a consequence of the particular means of determining activity or it could even conceivably be related to the possibility that fractions of different MW differ also with respect to composition.

Since molecular weight affects heparin biological activity, accurate determination of the molecular weight of heparin is important. However, classical techniques for MW determination are problematic for low-MW polyelectrolytes, which would permeate the membranes used for membrane osmometry, are not soluble in solvents appropriate for vapor phase osmometry, and are weak scatterers of light. ${ }^{13} \mathrm{C}$ NMR has been shown to provide reasonable MW values for heparin, but because it relies on quantization of the small number of end groups, the precision is not high even with very long acquisition times $[7,8]$. Therefore considerable effort has been directed toward size exclusion chromatography (SEC). ${ }^{1}$

\footnotetext{
${ }^{1}$ Abbreviations used: SEC, size exclusion chromatography; NaPSS, sodium poly(styrenesulfonate).
} 


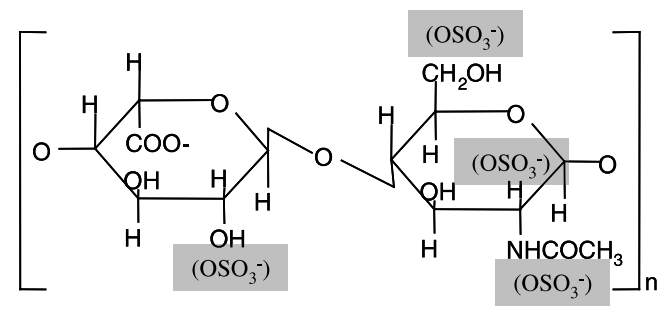

Heparin

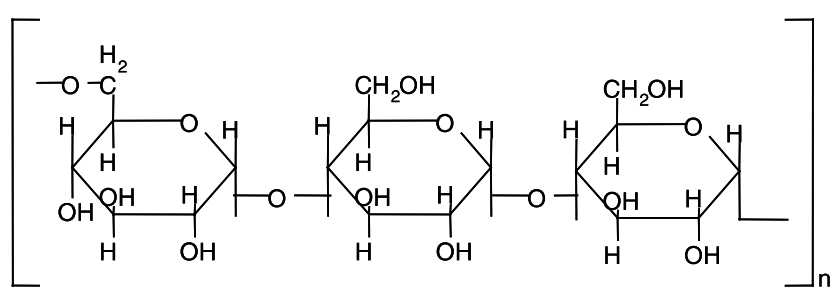

Pullulan

Fig. 1. Chemical structures of heparin and pullulan. The gray shading represents possible location of sulfonate groups.

Size exclusion chromatography, often referred to as gel permeation chromatography, is suitable for determining the molecular weight and molecular weight distribution (MWD) of polymers. Most rigorously, a set of fractionated standards with defined molecular weights and narrow MWDs are needed to calibrate the column. In the case of heparin, enzymatic degradation can lead to well-characterized oligosaccharides, but these standards would calibrate only the low-MW end of the distribution [9]. Higher-MW standards for heparin would be very costly to prepare and characterize at commercial levels. On the other hand, a universal calibration curve constructed by other polymer standards could provide a practical solution.

The concept of universal calibration was first introduced by Benoit and co-workers [10]. They demonstrated that a number of polymers with different configurations (e.g., linear, comb-branched, star-branched) all conformed to a single plot of $[\eta] M$ versus the elution volume $V_{\mathrm{e}}$, where $[\eta]$ denotes the intrinsic viscosity and $M$ is the weight average molecular weight [11]. This relationship is based on the Einstein viscosity law for spherical particles in suspension [12],

$[\eta]=v N \frac{V_{h}}{M}$,

and can be related to the Flory-Fox equation for linear polymers in solution [12],

$[\eta]=\Phi \frac{\left\langle s^{2}\right\rangle^{3 / 2}}{M}$,

where $v$ is a shape factor with a value of 2.5 for spheres, $V_{h}$ is the spherical volume, $N$ is Avogadro's number, $\Phi$ is a constant, and $\left\langle s^{2}\right\rangle^{1 / 2}$ is the root-mean-square radius of gyration of the polymer chain. According to both Eqs. (1) and (2), $[\eta] M$ is proportional to a kind of molecular volume. Although not rigorously defined, this molecular volume has been found to be universal for many polymers. Thus, a universal SEC curve can be constructed by plotting $[\eta] M$ versus elution volume.

Eq. (2) provides the relationship between measurable quantities and calculated dimensions of a statistical chain and is therefore limited to linear macromolecules with degrees of polymerization above ca. 50-100 [12], below which theories for the relationship between $[\eta]$ and $M$ are compromised. The theoretical basis for universal calibration in the limit of low MW is therefore uncertain. However, few exceptions to universal calibration in the low-MW range have been documented, and in fact universal calibration is seen for MWs in the range of $600-2000[13,14]$. In this low-MW range, the Mark-Houwink-Sakurada (MHS) relationship $[\eta]=$ $K M^{a}$, frequently employed in conjunction with universal calibration, cannot be used since the exponent $a$ becomes MW dependent, but this in itself does not invalidate the empirical finding that $[\eta] M$ can govern elution independent of polymer composition. Nevertheless, it has been suggested that universal calibration cannot be applied to heparin samples of different origin [15].

The universal calibration curve is most commonly obtained from and valid for neutral polymers. For proteins and polyelectrolytes, hydrophobic and electrostatic interactions may take place between the solute and the packing; most packings for aqueous SEC bear a negative surface charge, especially at neutral or high $\mathrm{pH}$ [16]. This calls into question the validity of universal calibration curves constructed with neutral standard polymer for heparin which is arguably one of the most highly charged biomacromolecules known. While it is known that electrostatic effects in SEC may be suppressed at high ionic strength, the remarkably large charge density of heparin raises the question of whether this can be practically achieved. The difficulty of suppressing such "polyelectroyte effects" might underlie the report that universal calibration fails for heparin and dextrans or polyethyleneglycols [15].

In this work, we establish the calibration curve with pullulan, a nonionic structurally well-defined polysaccharide (Fig. 1) available as narrow-MWD well-characterized MW standards [17,18]. To demonstrate the universality of this calibration, we also employ ficoll, a densely branched spheroidal nonionic polysaccharide $[19,20]$, and compare the elution of these two polymers to those of sodium poly(styrenesulfonate) (NaPSS), a polyelectrolyte frequently employed for SEC calibration. The validity of universal calibration for heparin is investigated at various ionic strengths using a sample characterized by light scattering and viscosity. 


\section{Experimental}

\section{Materials}

Pullulan Standards, Shodex P-82, were obtained from Showa Denko (Tokyo, Japan) with molecular weight and intrinsic viscosities listed in Table 1. Ficoll fractions were obtained from Dr. Rune Andersson of Pharmacia (Table 2). NaPSS samples with different molecular weights were purchased from Pressure Chemical (Pittsburgh, PA) (Table 3). Heparin sodium salt from porcine intestinal mucosa was purchased from Calbiochem (Lot B35123; nominal molecular weight supplied by manufacturer, 13,500-15,000 g/mol) (Table 4). DNA sodium salt from calf thymus was from Sigma (Lot 95H9526) and deuterium oxide $(99.9 \%)$ was from Aldrich. All samples were used directly without further purification.

Aqueous phosphate buffer solutions of $0.1,0.2,0.3$, $0.5,1.0$, and $2.0 \mathrm{M}$ ionic strengths with $\mathrm{pH}$ ca. 7.0 were prepared using sodium phosphate monobasic (Mallinckrodt Chemical) and sodium phosphate dibasic (Baker Chemical). Milli-Q water was used in all experiments.

\section{Viscosity}

Viscosity measurements were carried out at $25 \pm 0.1^{\circ} \mathrm{C}$ using the Viscosity Measuring Unit AVS 300 (Schott Geraete) consisting of a Ubbelohde-type capillary viscometer and an automatic pumping and timing system. All solvents and solutions were filtered through Whatman $0.2-\mu \mathrm{m}$ syringe filters. The intrinsic viscosities $[\eta]$ were determined by extrapolation to zero polymer concentration.

\section{Size exclusion chromatography}

Size exclusion chromatography was performed on an apparatus composed of a NSI-33R Milton Roy Minipump (Riviera Beach FL), a Rheodyne (Cotati, CA) Model 7010 injector with a 100- $\mu 1$ sample loop, a Gilson (Middleton, WI) $112 \mathrm{UV} / \mathrm{Vis}$ detector, and a Waters Associates-Millipore (Milton, MA) R401 Differential Refractometer, coupled to a Kipp and Zonen twochannel recorder. A Superose 12 HR 10/30 column from Pharmacia Biotech (Piscataway, NJ) was connected between the injector and the detectors. Flow rates were

Table 1

Characteristics of pullulan standards

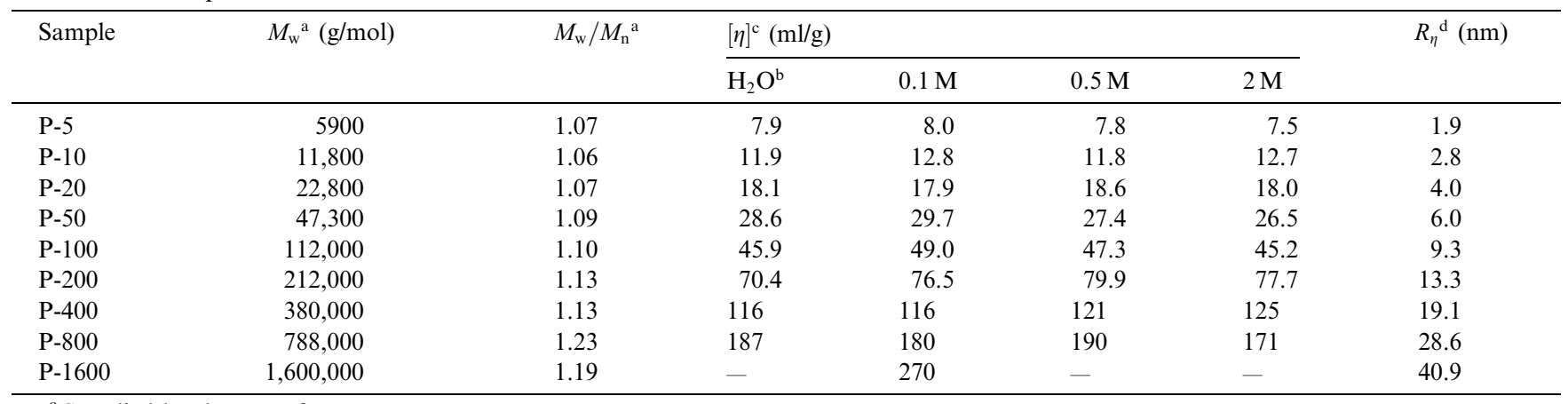

${ }^{\mathrm{a}}$ Supplied by the manufacturer.

${ }^{\mathrm{b}}$ In water at $25^{\circ} \mathrm{C}$ (supplied by the manufacturer).

${ }^{\mathrm{c}}$ Measured in phosphate buffer solution ( $\left.\mathrm{pH} 7.0\right)$ at $25^{\circ} \mathrm{C}$, with ionic strength shown.

${ }^{\mathrm{d}}$ Hydrodynamic radius in pure water calculated according to Eq. (3).

Table 2

Characteristics of ficoll fractions

\begin{tabular}{|c|c|c|c|c|c|c|c|}
\hline Sample & $M_{\mathrm{w}}{ }^{\mathrm{a}}(\mathrm{g} / \mathrm{mol})$ & $M_{\mathrm{w}} / M_{\mathrm{n}}{ }^{\mathrm{a}}$ & \multicolumn{4}{|c|}{$[\eta](\mathrm{ml} / \mathrm{g})$} & $R^{\mathrm{a}}(\mathrm{nm})$ \\
\hline F-30 & 21,292 & 1.22 & 6.8 & 7.0 & 7.0 & 7.0 & 3.0 \\
\hline F-46 & 60,710 & 1.15 & 9.4 & 10.5 & 10.7 & 10.5 & 4.6 \\
\hline F-60 & 105,209 & 1.13 & 12.2 & 12.5 & 12.4 & 12.4 & 6.0 \\
\hline F-73 & 175,527 & 1.20 & 13.3 & 14.9 & 15.7 & 15.1 & 7.3 \\
\hline
\end{tabular}

\footnotetext{
${ }^{\mathrm{a}}$ From supplier.

${ }^{\mathrm{b}}$ Measured in Milli-Q water at $25^{\circ} \mathrm{C}$.

${ }^{\mathrm{c}}$ Measured in phosphate buffer solution ( $\left.\mathrm{pH} 7.0\right)$ at $25^{\circ} \mathrm{C}$, with ionic strength shown.
} 
Table 3

Intrinsic viscosities of sodium poly(styrenesulfonate)

\begin{tabular}{cccccc}
\hline$M_{\mathrm{w}}{ }^{\mathrm{a}}(\mathrm{g} / \mathrm{mol})$ & {$[\eta]^{\mathrm{b}}(\mathrm{ml} / \mathrm{g})$} & & & \\
\cline { 2 - 5 } & $0.00115 \mathrm{M}$ & $0.1 \mathrm{M}$ & $0.2 \mathrm{M}$ & $0.3 \mathrm{M}$ & $0.5 \mathrm{M}$ \\
\hline 5400 & 11 & 4.1 & 3.9 & 3.5 & 3.2 \\
12,000 & 32 & 7.2 & 22 & 6.6 & 21 \\
35,200 & 120 & 32 & 30 & 28 & 25 \\
88,000 & 360 & & & 25 \\
\hline
\end{tabular}

\footnotetext{
${ }^{\mathrm{a}}$ Supplied by the manufacturer.

${ }^{\mathrm{b}}$ In solution with ionic strength shown. Calculated according to Mark-Houwink equation and an empirical relationship: $[\eta] \propto I^{-0.6}$ (drawn from data in [27, Table II]).
}

Table 4

Characterization of heparin

\begin{tabular}{lll}
\hline$I^{\mathrm{a}}(\mathrm{M})$ & {$[\eta](\mathrm{ml} / \mathrm{g})$} & $R_{\eta}{ }^{\mathrm{b}}(\mathrm{nm})$ \\
\hline 0 & 80.6 & 5.2 \\
0.1 & 19.8 & 3.3 \\
0.5 & 17.3 & 3.1 \\
1 & 16.9 & 3.1 \\
2 & 16.7 & 3.1 \\
\hline
\end{tabular}

${ }^{\mathrm{a}}$ Ionic strength of phosphate buffer (pH 7.0). All measurements performed at $25^{\circ} \mathrm{C}$.

${ }^{\mathrm{b}}$ Hydrodynamic radius calculated according to Eq. (3).

determined by weighing of timed collections of eluant and were reproducible to within $\pm 1 \%$. Chart recorder speed was usually $1 \mathrm{~cm} / \mathrm{min}$ and determined to within $\pm 1 \%$. All measurements were done at room temperature. Injections were performed in a mobile phase of Milli-Q water or buffers, which were filtered with 0.45 $\mu \mathrm{m}$ Acetate Plus filters (MSI, MA) and degassed before using.

\section{Static light scattering}

The lyophilized heparin sample was dissolved in $0.1 \mathrm{M} \mathrm{NaCl}$ to give a concentration of ca. $5 \mathrm{mg} / \mathrm{ml}$. The solution was centrifuged in a Beckman preparative ultracentrifuge Model L-70 for $90 \mathrm{~min}$ at $40,000 \mathrm{rpm}$ and $25^{\circ} \mathrm{C}$ using a fixed-angle rotor $\mathrm{Ti} 70.1$. The supernatant was filtered through a $0.2-\mu \mathrm{m}$ pore membrane filter (cellulose nitrate; Sartorius-Membranefilter $\mathrm{GmbH}$, Germany). A concentration series covering the range between about 1 and $5 \mathrm{mg} / \mathrm{ml}$ was filtered through a 0.1 $\mu \mathrm{m}$ pore size filter directly into the measuring cells. The precise polymer concentration was checked refractometrically, indicating that $97.5 \%$ of the original material was retained. The resultant concentrations were: 1.05 , $3.15,4.20$, and $5.25 \mathrm{mg} / \mathrm{ml}$. Light scattering measurements were performed at $25^{\circ} \mathrm{C}$ in a modified Sofica goniometer (Model 42000, FICA, France), with a laser light source of $\lambda_{\mathrm{o}}=632 \mathrm{~nm}$. The angle of observation was varied between $30^{\circ}$ and $145^{\circ}$ in steps of $5^{\circ}$. The specific refractive index increment $\left(\lambda_{\mathrm{n}} / \lambda_{\mathrm{c}}=0.135 \mathrm{ml} / \mathrm{g}\right)$, which was used for the Zimm procedure, was deter-

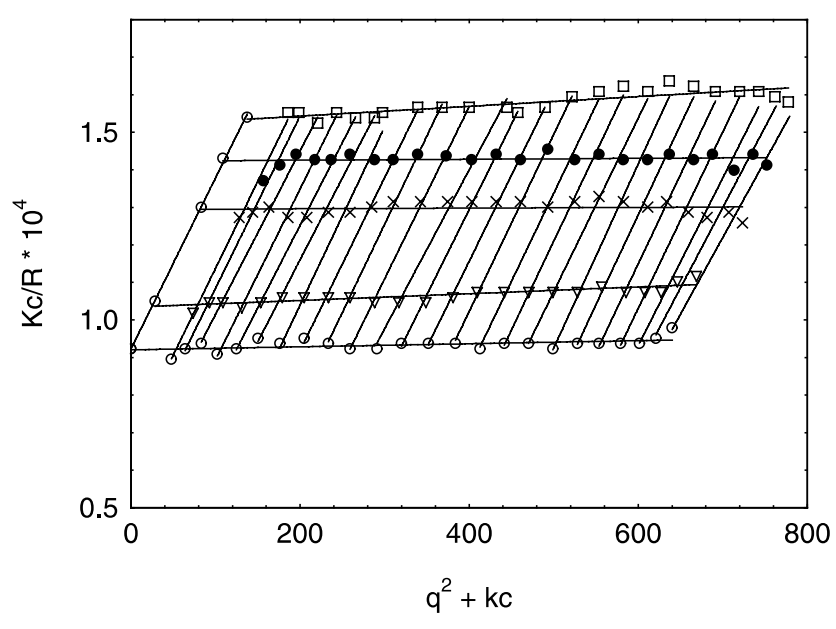

Fig. 2. Zimm plot of heparin sample.

mined at $\lambda=632 \mathrm{~nm}$ using an interferometric differential refractometer (ScanRef, Nanofilm Technology, Germany) at $25.0^{\circ} \mathrm{C}$.

The weight-average molecular weight of the heparin sample was determined as $10,900 \mathrm{~g} / \mathrm{mol}$ by static light scattering (see Zimm plot in Fig. 2). The radius of gyration and the second virial coefficient $A_{2}$ were $11.5 \mathrm{~nm}$ and $5.9 \times 10^{-6} \mathrm{molL} / \mathrm{g}^{2}$, respectively, although the former must be considered a rough estimate.

\section{Results}

\section{Intrinsic viscosities}

As pullulan is a neutral polymer, its intrinsic viscosity should be insensitive to ionic strength I. Intrinsic viscosities of pullulan samples in $0.1,0.5$, and $2 \mathrm{M}$ phosphate buffer solutions in Table 1 confirm this weak dependence. Within experimental error, the data at all ionic strengths conform to a single Mark-HouwinkSakurada (MHS) plot (Fig. 3), $[\eta]=0.0276 M^{0.65}$, the value of the exponent being identical to that for literature results for pure water $[17,18]$. It should be noted that a $10 \%$ change of $[\eta]$ and its effect on $\log ([\eta] M)$ will 


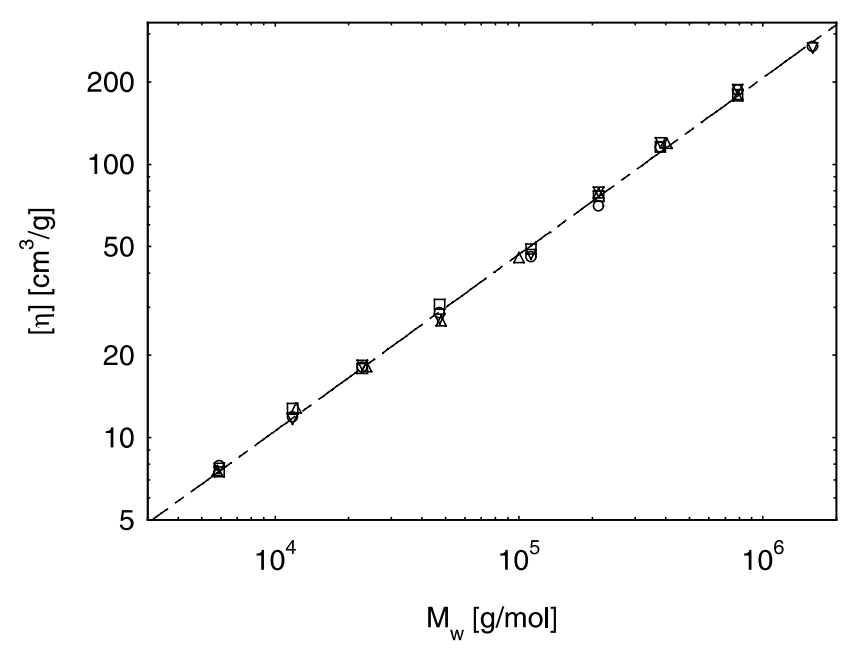

Fig. 3. MHS plots for pullulan at varying ionic strengths (data in Table 1): ( $\bigcirc$ ) in Milli-Q water; $(\square)$ in $0.1 \mathrm{M}$ phosphate buffer solution; $(\nabla)$ in $0.5 \mathrm{M}$ phosphate buffer solution; $(\triangle)$ in $2 \mathrm{M}$ phosphate buffer solution.

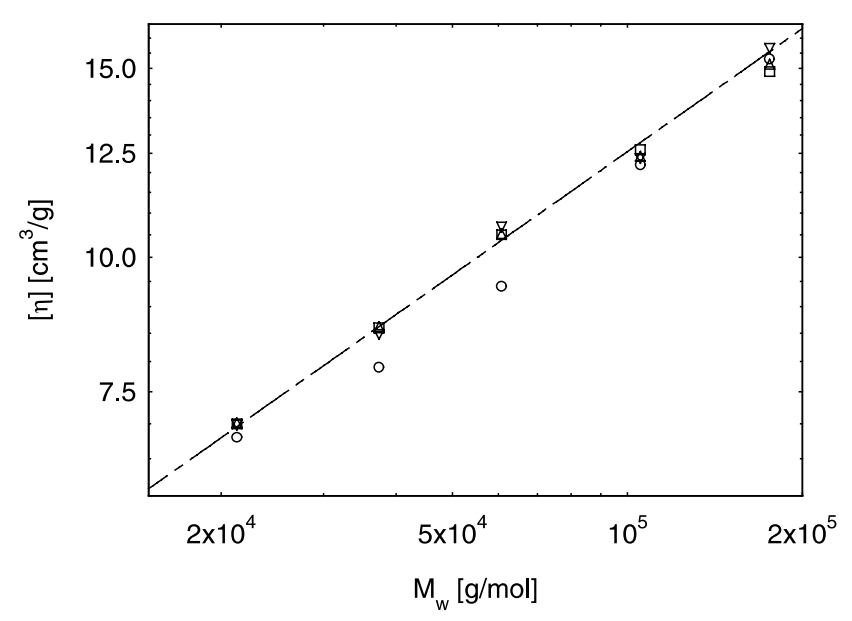

Fig. 4. MHS plots for ficoll fractions at varying ionic strengths (data in Table 2): (O) in Milli-Q water; $(\square)$ in $0.1 \mathrm{M}$ phosphate buffer solution; $(\nabla)$ in $0.5 \mathrm{M}$ phosphate buffer solution; $(\triangle)$ in $2 \mathrm{M}$ phosphate buffer solution.

have a minimal impact on the universal calibration curve.

Ficoll, generally considered to be nonionic, possesses a small amount of negative charge [21,22]. Intrinsic viscosities at $0<I<2 \mathrm{M}$, recorded in Table 2, lead to the plot of Fig. 4. The corresponding MHS equation $[\eta]=0.156 M^{0.38}$ is in good agreement with the value of $a=0.35$ reported elsewhere [20], although the data in pure water deviate. It seems that $0.1 \mathrm{M}$ ionic strength is enough to suppress electrostatic effects on $[\eta]$.

For heparin in pure water, $\eta_{\mathrm{sp}} / c$ rises with decreasing concentration due to the expansion of the polyelectrolyte. The intrinsic viscosity was determined from the intercept $(1 /[\eta])$ at $c^{1 / 2} \rightarrow 0$ as suggested by Fuoss and Strauss [23].

\section{Universal calibration curve}

A universal calibration curve (Fig. 5) based on the measurement of the elution volumes of standard pullulan samples and ficoll fractions in solutions with different ionic strength was constructed. The elution volumes of pullulan and ficoll were found to increase slightly as the ionic strength of the phosphate buffer increased; the universal calibration curve shifts slightly to the right. As the intrinsic viscosities for both pullulan and ficoll are almost invariant with ionic strength in the chosen buffer solutions, the change of solute size should be negligible. Therefore, the shift of curves is presumably due to changes in the pore size of the stationary phase $[21,24]$. $\mathrm{D}_{2} \mathrm{O}$ and DNA were used to measure total column liquid volume, $V_{\mathrm{t}}$, and interstitial volume, $V_{0}$, respectively. In pure water, $V_{\mathrm{t}}=20.9 \mathrm{ml}$ and $V_{0}=7.9 \mathrm{ml}$, but changed to $V_{\mathrm{t}}=21.3 \mathrm{ml}$ and $V_{0}=7.7 \mathrm{ml}$ in $0.5 \mathrm{M}$ phosphate buffer solution, corresponding to an increase of ca. $4 \%$ in $V_{\mathrm{t}}-V_{0}$, the overall pore volume. This increase of pore size leads to the shift of calibration curves toward higher $V_{\mathrm{e}}$ with increasing ionic strength which has been previously observed [21] and interpreted [24].

The negative charge of ficoll fractions causes a strong decrease in the elution volumes in pure water (data not shown). The effect is fully suppressed when $I \geqslant 0.1 \mathrm{M}$. Aqueous size-exclusion chromatography of NaPSS has been investigated intensely [25-27], including studies of universal calibration. As shown in Fig. 6, all NaPSS samples eluted in pure water at the interstitial volume $V_{0}$, regardless of MW. Retention volumes of NaPSS increased with increasing ionic strength, but under no condition would the data for NaPSS coincide with the universal calibration curve, diverging from it in both

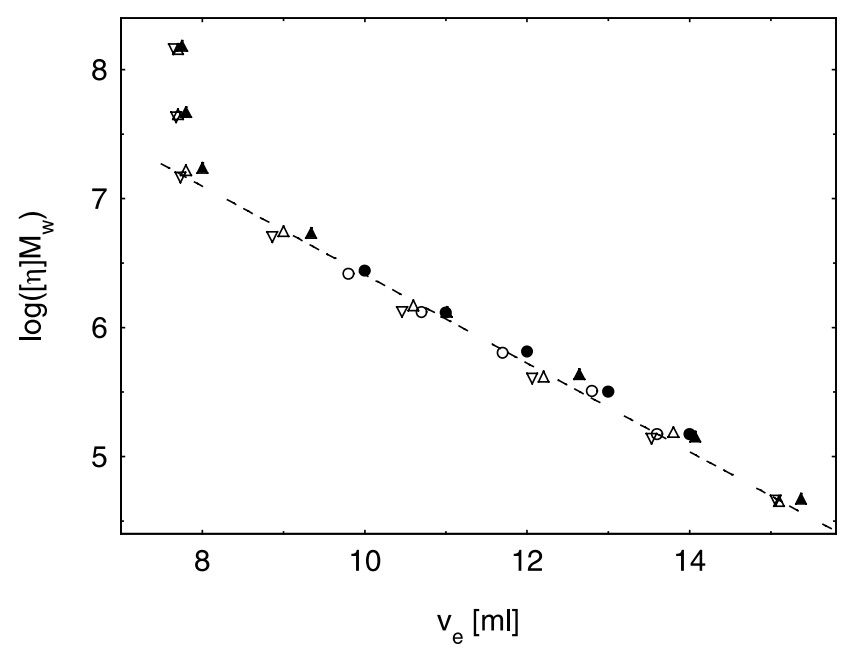

Fig. 5. Universal calibration plot for standard pullulan samples and ficoll fractions: $(\nabla)$ Pullulan in Milli-Q water; $(\triangle)$ pullulan in $0.1 \mathrm{M}$ phosphate buffer solution; ( $\boldsymbol{\Delta})$ pullulan in $0.5 \mathrm{M}$ phosphate buffer solution; ( $\bigcirc$ ) ficoll fractions in $0.1 \mathrm{M}$ phosphate buffer solution; ficoll fractions in $0.5 \mathrm{M}$ phosphate buffer solution. 


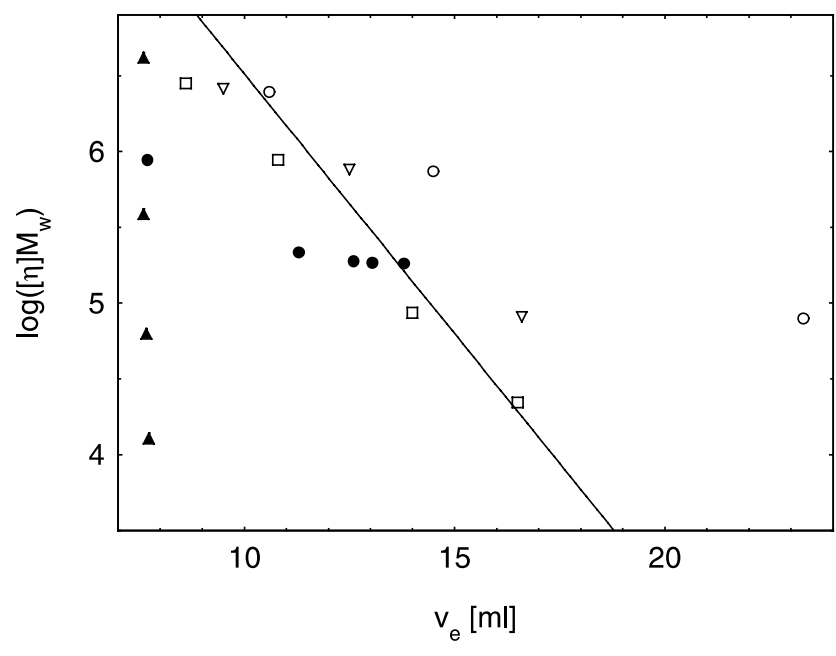

Fig. 6. Location of NaPSS and heparin around the universal calibration curve (data for pullulan in $0.5 \mathrm{M}$ phosphate buffer solution): $(\mathbf{\Delta})$ NaPSS in Milli-Q water; ( $\square$ ) NaPSS in $0.1 \mathrm{M}$ phosphate buffer solution; $(\nabla) \mathrm{NaPSS}$ in $0.2 \mathrm{M}$ phosphate buffer solution; $(\bigcirc)$ NaPSS in $0.3 \mathrm{M}$ phosphate buffer solution; $(\bullet)$ represents heparin in Milli-Q water and in $0.1,0.5,1$, and $2 \mathrm{M}$ phosphate buffer solution, from left to right.

directions depending on ionic strength. This behavior arises from concomitant effects of repulsion and hydrophobic interactions, the latter increasing with $I$, the former decreasing with $I$. Similar results were obtained by Mori [25].

\section{Heparin}

As seen in Fig. 6, the elution volume of heparin increased with ionic strength $(I)$, converging on the universal calibration curve within experimental error at $I=2 \mathrm{M}$. Effective screening of electrostatic repulsion by increasing the salt concentration apparently results in universal calibration with heparin.

\section{Discussion}

The viscosity radius $R_{\eta}$ of heparin (see Table 4 ) can be calculated from [12]:

$R_{\eta}=\left(\frac{3[\eta] M}{10 \pi N_{\mathrm{A}}}\right)^{1 / 3}$.

An apparent SEC radius $R_{\mathrm{SEC}}$ is defined from the universal calibration curve $\left(R_{\eta}\right.$ versus $\left.V_{\mathrm{e}}\right)$ constructed from pullulan data. The difference between $R_{\mathrm{SEC}}$ and $R_{\eta}$ $\left(\Delta R=R_{\mathrm{SEC}}-R_{\eta}\right)$ quantitatively represents the effect of electrostatic repulsion between solute and matrix [22]. Fig. 7 depicts the elution volume and $\Delta R$ as a function of ionic strength. Effective screening of electrostatic interaction for heparin can be achieved only when $I>1.0 \mathrm{M}$. For SEC subject to solute-packing electrostatic repulsion, Potschka suggested [28-30],

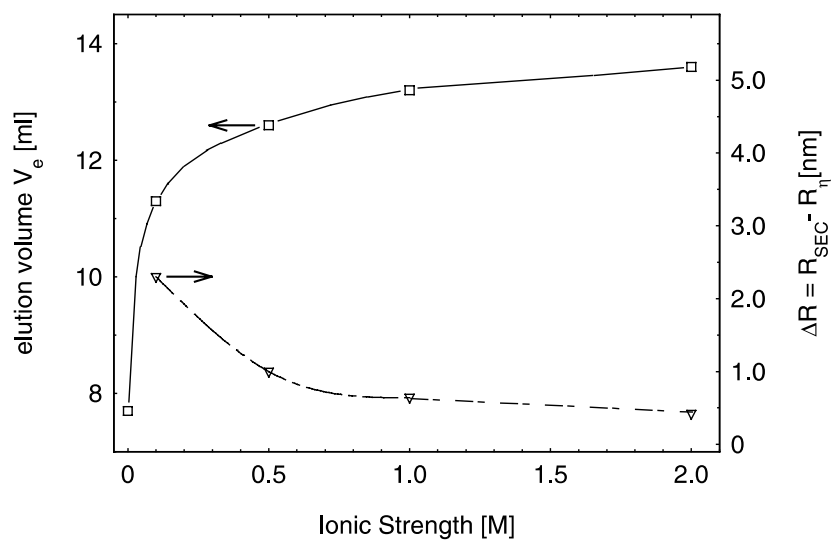

Fig. 7. Elution volume and $\Delta R=R_{\mathrm{SEC}}-R_{\eta}$ of heparin as a function of ionic strength. Lines are to guide the eye.

$R_{\mathrm{SEC}}=R_{\eta}+\kappa^{-1} x \cong R_{\eta}+0.3 I^{-1 / 2} x$,

where $\kappa^{-1}$ is the Debye length and $x$ is the average electrostatic repulsion distance at equilibrium in multiples of Debye lengths. If the apparent elution size is plotted versus the Debye length of the mobile phase, a linear relationship should be obtained with a slope of $x$. According to Potschka, $x$ depends on the size of the solute and the matrix charge and is approximately proportional to the average net charge of the solute [21]. The slope in Fig. 8 of 2.7 is large compared to values seen for myoglobin (horse) (0.57), ovalbumin (2.08), and $\beta$-lactoglobulin (1.74) [21], indicative of heparin's high charge density.

An approximation of the polydispersity of the heparin sample is provided by reference to the pullulan calibration curve, giving $\left(M_{\mathrm{w}} / M_{\mathrm{n}}\right)_{\text {pul }}=1.2$. An accurate value of $\left(M_{\mathrm{w}} / M_{\mathrm{n}}\right)$ can be obtained only with the true $M$ vs $V_{\mathrm{e}}$ calibration curve for heparin. In principle, this could be obtained even with polydispersed samples of

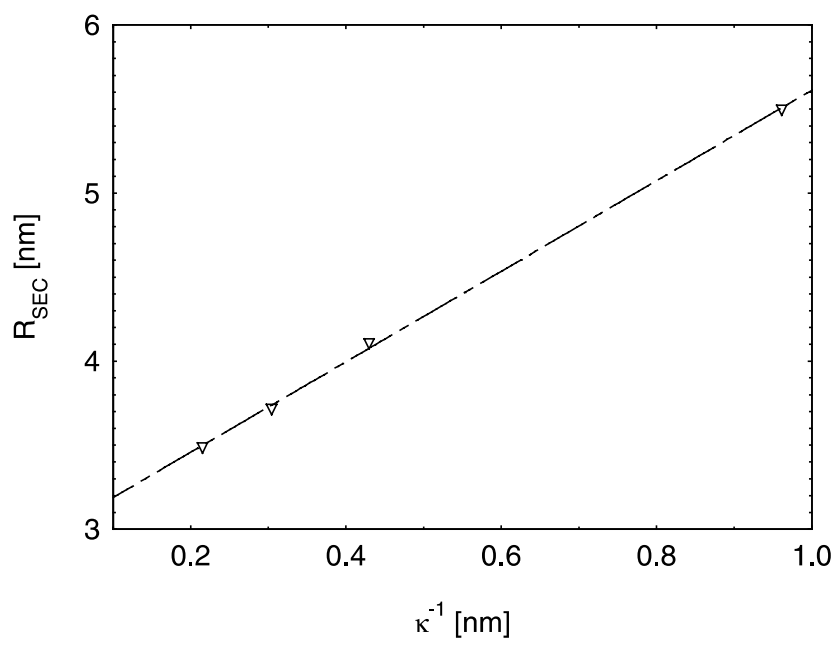

Fig. 8. Apparent SEC radii as a function of Debye length $\left(\kappa^{-1}=\right.$ $\left.0.304 I^{-1 / 2}\right)$. 
known $M_{\mathrm{w}}$ or $M_{\mathrm{n}}$ by an iterative procedure [31], but such samples were not available in the present study.

For determination of the molecular weight of a heparin sample using this method, either of two approaches may be followed. In principle, if the molecular weight is large enough $\left(>10^{4}\right)$ and the MHS relationship is well defined, the molecular weight can be easily calculated via $[\eta] M=K M^{a+1}$, where $[\eta] M$ is obtained directly from the universal calibration curve after measurement of the elution volume, with literature values of $K$ and $a$. Such values are not well established for heparin, and the MHS relationship itself may be invalid at such low MW. Nevertheless, the molecular weight can be determined from the value of $[\eta] M$ and intrinsic viscosity measured in the SEC eluant. If the intrinsic viscosity can be measured as here with a precision of $\pm 0.4 \%$, then the error in MW arises from uncertainty in the elution volume typically around $\pm 0.5 \%$. The propagated uncertainty for the molecular weight is then around $4 \%$.

\section{Conclusion}

The dependence of $\log \left([\eta] M_{\mathrm{w}}\right)$ on $V_{\mathrm{e}}$ for standard pullulan samples in solutions with any ionic strength can serve as a universal calibration curve. Because ficoll fractions contain negative charges, they can be used to construct the universal curve only after repulsion effects are screened in an electrolyte solution with $I>1.0 \mathrm{M}$. Universal calibration with NaPSS fails because of repulsive interaction between NaPSS and packing materials at low $I$ and because of adsorption at high $I$. The elution volume of heparin is strongly dependent on $I$. Universal calibration can be used to accurately determine the molecular weight of heparin when the electrostatic interaction is totally screened by high ionic strength $(I=2 \mathrm{M})$. This ionic strength is large compared to that required to suppress repulsion on even more highly charged packings [16] and is a reflection of the extraordinarily high charge of heparin.

\section{Acknowledgment}

Support from NSF Grant CHE-9987891 is acknowledged.

\section{References}

[1] S. Monique, A. Kher, F. Toulemonde (Eds.), Low Molecular Weight Heparin Therapy-An Evaluation of Clinical Trials Evidence, Marcel Dekker, New York, 1999, p. 12.

[2] B. Casu, Pharmacol. Res. Commun. 11 (1) (1979) 1.

[3] B.A. Khorramian, S.S. Stivalla, Arch. Biochem. Biophys. 247 (2) (1986) 384.

[4] C. Doutremepuich (Ed.), Low Molecular Weight Heparin in Clinical Practice, Marcel Dekker, New York, 1992, p. 7.

[5] H.J. Rodriguez, A.J. VanDerWielen, J. Pharm. Sci. 68 (5) (1979) 588.

[6] B. Mulloy, E. Gray, T.W. Barrowcliffe, Thrombosis Haemostasis 84 (6) (2000) 1052

[7] U.R. Desai, R.J. Linhardt, Carbohydr. Res. 255 (1994) 193.

[8] U.R. Desai, R.J. Linhardt, J. Pharm. Sci. 84 (1995) 212.

[9] A. Ahsan, W. Jeske, H. Wolf, J. Fareed, Clin. Appl. Thrombosis/ Hemostasis 6 (3) (2000) 169.

[10] H. Benoit, Z. Grubisic, P. Rempp, D. Decker, J.G. Zilliox, J. Chem. Phys. 63 (1966) 1507.

[11] Z. Grubisic, Rempp, H. Benoit, Polym. Lett. 5 (1967) 753.

[12] P.J. Flory, in: Principles of Polymer Chemistry, Cornell University Press, Ithaca New York, 1953, p. 606, see also p. 611.

[13] L. Mrikvickova, J. Liq. Chromatogr. Rel. Technol. 20 (1997) 403.

[14] C. Tsitsilianis, G. Mitsiani, A. Dondos, J. Polym. Sci. Part B: Polym. Phys. 27 (1989) 763.

[15] W.E. Hennink, J.W.A. Van den Berg, J. Feijen, Thromb. Res. 45 (1987) 463.

[16] P.L. Dubin (Ed.), Aqueous Size-exclusion Chromatography, Elsevier Science Publishers, New York, 1988, p. 10, see also p. 60,66 .

[17] K. Nishinari, K. Kohyama, P.A. Williams, G.O. Phillips, W. Burchard, K. Ogino, Macromolecules 24 (1991) 5590.

[18] G.S. Buliga, D.A. David, Int. Biol. Macromol. 9 (1987) 71

[19] M.G. Davidson, W.M. Deen, Macromolecules 21 (1988) 3474.

[20] P.N. Lavrenko, O.I. Mikryukova, S.A. Didenko, Polym. Sci. USSR 28 (1986) 576.

[21] Y. Zhu, M. Potschka, P.L. Dubin, C. Cai, Macromol. Chem. Phys. 202 (2001) 61.

[22] Y. Wang, P.L. Dubin, J. Chromatogr. A 800 (1998) 181.

[23] R.M. Fuoss, U.P. Strauss, J. Polym. Sci. 3 (1948) 602.

[24] M. Potschka, J. Chromatogr. 441 (1988) 239.

[25] S. Mori, Anal. Chem. 61 (1989) 530.

[26] P.L. Dubin, C.M. Speck, J.I. Kaplan, Anal. Chem. 60 (1988) 895.

[27] P.L. Dubin, M.M. Tecklenburg, Anal. Chem. 57 (1985) 275.

[28] M. Potschka, Macromolecules 24 (1991) 5023.

[29] M. Potschka, P.L. Dubin (Eds.), Strategies in Size Exclusion Chromatography, ACS Symposium Series, 635, American Chemical Society, Washington DC, 1996, p. 67.

[30] M. Potschka, J. Chromatogr. 587 (1991) 276.

[31] P.L. Dubin, K.L. Wright, S.W. Koontz, J. Polym. Sci. Polym. Chem. Ed. 15 (1977) 2047. 